\title{
Application of NSGA-II Algorithm to Multiobjective Optimization of Switching Devices Placement in Electric Power Distribution Systems
}

\author{
António Vieira Pombo ${ }^{1,2}$, Vitor Fernão Pires ${ }^{1,2}$, and João Murta Pina ${ }^{1}$ \\ ${ }^{1}$ Faculdade de Ciências e Tecnologia \\ Universidade Nova de Lisboa \\ Monte da Caparica, Portugal \\ jmmpafct.unl.pt \\ ${ }^{2}$ Escola Superior de Tecnologia \\ Instituto Politécnico de Setúbal \\ Setúbal, Portugal \\ \{antonio.pombo,victor.pires\} @estsetubal.ips.pt
}

\begin{abstract}
The Electric Utility Industry all around the world is facing numerous challenges, which include amongst others, the optimal use of expensive assets and resources and the maintenance of electric grid and customer quality service levels. The optimal placement of switches in electrical distribution networks will allow the control over service quality levels and the maximization of investments in equipments. This work proposes a genetic evolutionary algorithm NSGA-II for the optimization between the maximal return of investments on existing assets, while maintaining the quality of service provided. The trade off between total cost of investments and service quality levels SAIDI (System Average Interruption Duration Index) and SAIFI (System Average Interruption Frequency Index) is analyzed, to choose the optimal placement of switches in the distribution electrical networks. The proposed method was tested with a Portuguese real distribution network. The obtained results allowed to verify the performance of the adopted approach.
\end{abstract}

Keywords: Switch Placement, Reliability, Genetic Algorithm, Electrical Distribution Networks.

\section{Introduction}

Planning and operation in Distribution system consists on satisfying the system load and energy demand, as economically as possible, while assuring supply continuity and contracted quality levels. Normally, the approaches that public owned utilities tend to implement consists on designing and operating electrical distribution infrastructures to minimize the total cost to the society. Generally this approach is referred to as value based planning.

On the other hand, private owned utilities tend to defer in time network investments and maximize their return. Their goal consists on supplying safe and reliable electric 
power at a reasonable cost. Recent trends however show that the future focus will not be only on the companies' economic performance, but rather on successfully combining economic performance with business impacts on social welfare and environmental factors (Fig. 1). Aging infrastructures and reliability are the most critical issues that preoccupy utility CEOs. And these two are closely related. A decrease in infrastructure investment decreases in time the reliability of the network. On the other hand an increase in network investment increases system reliability. But why focus on reliability? The effects of power outages go beyond the inconvenience experienced from the outage itself. Power outages cost local businesses thousands of euros in lost sales, interrupted manufacturing and lost data. The economic cost of outages reaches beyond lost productivity. A multi-day outage can cost residents hundreds of dollars in lost food and can damage other personal items when sump pumps stop working. Under this context, in electric power distribution system planning, the optimal placement of switches is one of the areas that contribute to the utility goal achievement of operating in the social welfare area. By choosing the optimal place and number of switches in the distribution electrical network, it is possible to maximize the quotient between the benefits to the community and the investments made by the utility on the network. By changing the state of the switches (open/close) the distribution network can be reconfigured, after a fault, thus minimizing the number of customers affected, fault duration and frequency. Although with different objectives, all reconfiguration problems are a combinatorial constrained problem, described by a nonlinear and non-differential objective function.

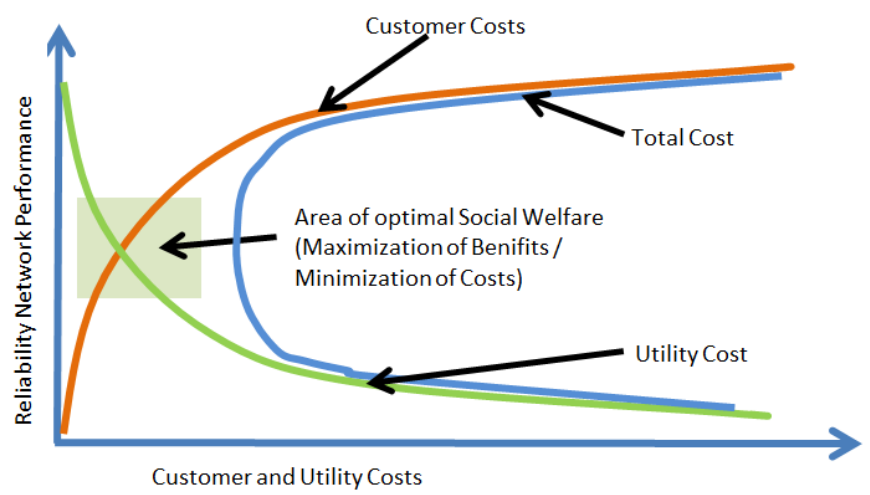

Fig. 1. Value Based Planning and Social Welfare operation

To address this optimization problem, where the objectives are generally conflicting, preventing simultaneous optimization of each objective, several algorithms have been developed. Due to the importance of the problem, several research articles related to optimal placement of switches in distribution networks have been written. In these works, different mathematical models and optimization methodologies have been used. Some of them present this issue as a single objective problem. Others use several objectives functions, but they are agglomerated into a new single objective function. Finally, some authors deal with this problem with 
several objective functions that are not agglomerated, allowing in this way to obtain a Pareto Optimality. Several optimization methods have been used to address the optimal switch placement problem such as Genetic Algorithms (GA) [1], [2], Particle Swarm Optimization (PSO) [3], [4], Ant Colony Systems (ACS) [5], [6] and Tabu Search Algorithm (TSA) [7]. In [8] is used a modified shuffled frog leaping algorithm and in [9] a hybrid algorithm, both using a multi-objective fuzzy logic (FL) approach.

In this work, a new algorithm applied to this problem is proposed. Due to the characteristics of this kind of problem, two important reliability measures generally used by electric utilities were selected for the objective functions [10]. These are SAIFI, which is an indicator of utility network performance and SAIDI, which measures the operating performance of the utility in restoring customer interruptions. The evolutive genetic algorithm NSGA-II was adopted for the optimization of this problem. This kind of genetic Algorithm is a popular meta-heuristic algorithm that have been applied to multiobjective optimization in power systems such as environmental/economic power dispatch [11] and capacitor placement [12], and as can be seen by this work is well suited for this type of problem. In this multi-objective optimization the goal is not to find a single optimal solution, but rather a trade off between different objective functions, called the "Pareto Set", which is the set of all feasible solutions whose vector of the various objectives is not dominated by any other solution. Results of a real distribution feeder are presented in order to verify the effectiveness of the proposed approach.

\section{Contribution to the Technological Innovation for Collective Awareness Systems}

This paper considers the methodology for best placing switches in a electrical distribution network, taking into account the information ("internet of things") of existing distribution structures, equipments, number of customers, etc., so that the decision taken has a collective awareness of the systems in witch it is going to have an effect. This awareness is manifested in an innovation of the criteria's behind the methodology for switch placement.

On the other hand, the implications of the placement and operation of switches in the distribution network, will provide information to higher systems, so that they too have an awareness of this system and in that measure also take an innovative perspective on other solutions affecting the planning and operation of electrical distribution networks.

\section{Problem Formulation}

As said before, in multi-objective optimization we are not interested in finding a single optimal solution, but rather a trade off between the two different objective functions, called the "Pareto Set", which is the set of all feasible solutions whose vector of the various objectives is not dominated by any other solution. Considering 
that which preoccupies most the CEOs of electric utilities is aging infrastructures and reliability, we chose system reliability (SAIDI and SAIFI) [10] and investment in equipment and installation costs for the objective functions to be minimized.

SAIDI (System Average Interruption Duration Index) is considered one the most widely spread indices used by electrical utilities to measure reliability. The mathematical representation of this index is presented below:

$$
S A I D I=\frac{\sum r_{i} N_{i}}{N_{t}}
$$

where:

$r_{i}$ is the average outage time per interruption of load point $i$ due to outages in section $s$;

$N_{i}$ is the number of clients in load point $i$;

$N_{t}$ is the total number of clients in the network;

Another reliability system index that is used is the SAIFI (System Average Interruption Frequency Index). The difference between this index and the previous one (SAIDI), is that instead of considering the average interruption duration, in this index is considered the average interruption frequency. Equation (2) presents the mathematical representation of the SAIFI where $\lambda_{i}$ is the average outage time per interruption of load point $\mathrm{i}$ due to outages in section s. Comparing equation (1) and (2) it is possible to verify that the difference variables $r_{i}$ and $\lambda_{i}$. However $r_{i}$ represents interruption time and $\lambda_{i}$ represents interruption frequency, which gives a completely different concept.

$$
S A I F I=\frac{\sum \lambda_{i} N_{i}}{N_{t}}
$$

Beside the minimization of the reliability indexes SAIDI and SAIFI, a third minimization function is considered. This last function is the Total Costs (TC) in which is considered the investment in buying and installation of equipment cost associated with each solution for a given reliability (3).

$$
\mathrm{TC}=(\text { Equipment Cost })+(\text { Installation Cost })
$$

where:

Equipment Cost is the cost of switches;

Installation Cost is the cost of the installations of switches in the network;

A radial electrical distribution networks characterized by several feeders coming out of a substation and its branches was considered in this work.

The GA used to generate the "Pareto Set" of feasible solutions uses a binary representation representing the place of the switches in each branch. 


\section{The NSGA-II Genetic Algorithm}

The chosen Genetic Algorithm NSGA-II (Fast Non-dominated Sorting Genetic Algorithm) is an elitist multiobjective evolutionary algorithm (MOEA). This algorithm it is characterized by a fast nondominated sorting and by an efficient crowding-distance assignment approach. Due to its characteristics, it has been used in many applications and its performance tested in many comparative studies.

It consists on finding a vector " $x$ " that minimizes a given set of " $n$ " objective functions. The solutions are normally restricted by a series of constrains and consists on a set of results that satisfies the objective at an acceptable level without being dominated by any other solution. In this case, a solution is said to be Pareto Optimal solution and each solution cannot be improved with respect to any other objective without worsening at least one other objective.

The algorithm NSGA-II achieves the three conflicting goals expected for a GA, that is, the best known Pareto Front: 1) should be as close as possible to the true Pareto Front; 2) should be uniformly distributed and diverse; 3) should capture the all spectrum of the Pareto Front.

The general solving procedure of a GA is as following:

1. Generation of the initial $N$ population, each of which is randomly initialized. Each solution of the population is called a Chromosome;

2. Generation of new $Q$ solutions from existing ones, using two operators: Crossover and Mutation. In Crossover two chromosomes, called Parents are combined together to form a new chromosome, called offspring. The selection of the Parents considers the preference towards fitness values. Mutation introduces random changes and is applied at gene level. Typically the probability of this change is very small, less then $1 \%$. Crossover leads to the convergence of the population and mutation reintroduces genetic diversity to the population.

3. Evaluation and assignment of a fitness value to each solution;

4. Selection of $\mathrm{N}$ solutions from the $\mathrm{Q}$ set of solutions based on their fitness;

5. Continuation of the anterior four steps until the stopping criteria is met.

The fitness assignment function of the algorithm uses the Crowding Distance approach. This concept aims to obtain a uniform spread of solutions along the bestknown Pareto Front without using a fitness sharing parameter. This is done by: 1) ranking the population and identify non-dominated fronts; 2) for each objective function sort the solutions in the ascending order; 3) finding the total crowding distance of a solution. The main advantage of this approach is that a measure of population density around a solution is compounded without requiring a user-defined parameter. Considering two random solutions, if they are in the same non-dominated front, the solutions with a higher crowding distance wins, otherwise, the solution with the lowest rank is selected. 
The problem of Elitism, (the best solution found so far during the search has immunity against selection and always survives in the next generation), is obtained by the NSGA-II algorithm, using a fixed size $N$ population. In step 2 of the general solving procedure, the generation of the $Q$ solutions, considers the offspring solutions and the parent solutions. The fitness assignment considers the two populations and this makes sure that all the non-dominated solutions are included in the next populations $N$ and the selection based on the crowding distance will promote diversity.

Regarding the genetic encoding, a matrix with $N$ lines and $M$ columns is used. The lines of the matrix represent the population. The branches are numerated in a sequential form. Thus, the index of each column is directly related with the beginning of a branch. Inside on each element of the matrix it is used a binary codification, where 1 represents the allocation of a switch and 0 a branch without a switch in the beginning.

\section{Case Study}

In order to verify the effectiveness of the adopted algorithm, tests over a real case were implemented. Fig. 3 shows the 94-Bus Portuguese distribution feeder that was used in this work. This is a $15 \mathrm{kV}$ radial network with 94-bus feeder.

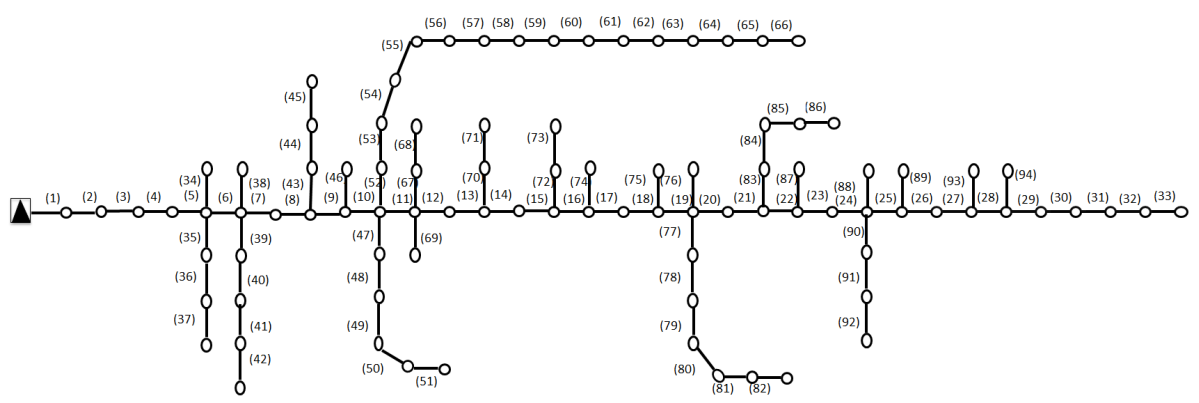

Fig. 2. Distribution network example for network representation

In order to evaluate the effectiveness of the proposed approach, two different tests have been made. SAIFI versus Cost was used as objective function in the first one. For the switch cost it was considered $2500 €$ and for the installation $1000 €$. 

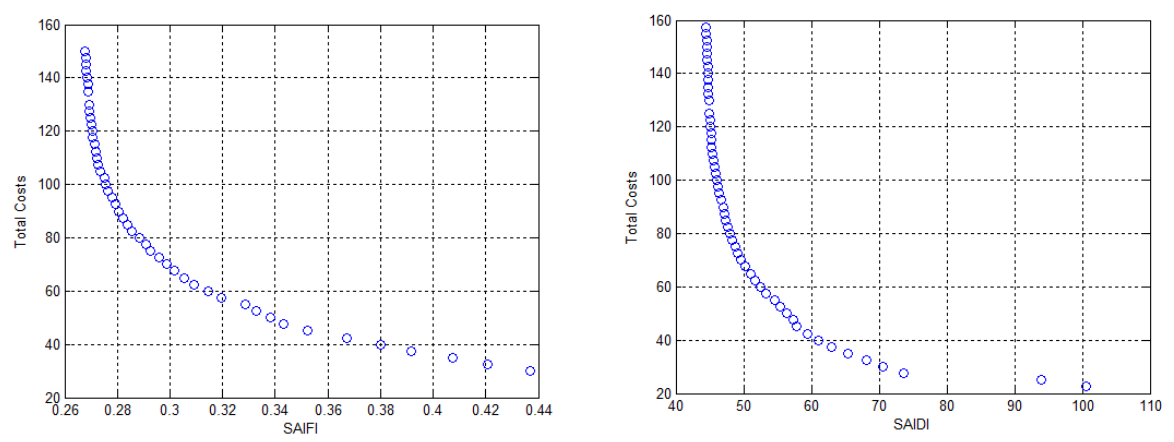

Fig. 3. Nondominated solutions obtained from de NSGA II algorithm considering as objective functions - Total Costs vs. SAIDI and Total Costs vs. SAIFI

The output of the NSGA II algorithm is presented in Fig. 4. In the figure the total costs is given in thousands of euros. As can be seen by the output of the algorithm, the final set of solutions is represented by the Pareto front. Each solution of the Pareto front represents a specific solution that is optimal in its own way. In fact, from these results it is possible to verify that when the costs improves the SAIFI decreases and vice-versa. The decision maker can select a particular solution from these multiple solutions. Table 1 shows the number and location of the switches for the best solution considering only SAIFI and the best solution considering only costs. As expected, for the SAIFI best solution it is required a huge number of switches, 60 . However, for the SAIFI worst solution (and best of the costs) the number of switches is much reduced, 12 .

Table 1. Number and location of the switches for nondominated solutions

\begin{tabular}{ccc}
\hline SAIFI & $\begin{array}{c}\text { COSTS } \\
\text { (thousand(€) })\end{array}$ & LOCATION OF THE SWITCHES \\
\hline 0.27 & 150 & $2,4,6,7,8,9,10,11,12,13,15,16,19,20,21,23,25,29,30,31,33,34,37$, \\
& & $38,40,42,45,46,48,50,51,52,53,54,55,56,57,58,60,61,62,63,64,65$ \\
& & $67,68,70,72,73,74,75,76,80,81,85,86,87,88,91,92$ \\
0.44 & 30 & $6,11,20,34,35,39,43,46,47,55,77,89$ \\
\hline
\end{tabular}

For the second test SAIDI versos cost was used as objective function. Fig. 4 shows the output of the algorithm using this new objective function. As expected when the cost improves the SAIDI decreases and vice-versa.

\section{Conclusions}

The allocation of switches in an electric distribution network represents an important measure to improve the power quality. Under this context, this paper presents a multi objective optimization technique to determine the optimal number and place of switches in an electrical distribution feeder. Reliability index SAIDI and SAIFI and 
cost of equipment's are the objective functions that are minimized to obtain the number and location of switches in an electrical distribution feeder. The algorithm used to find the optimal placement of switches is a Genetic Algorithm NSGA-II. The multi objective problem uses the Pareto optimal front to obtain the set of non dominated solutions. The adopted algorithm was applied to a 94-Bus Portuguese network to illustrate its performance. The number and location of switches in the distribution feeder have been determined in order to minimize the equipments cost and the reliability indexes SAIDI and SAIFI. This algorithm also shows the advantage of multiobjective optimization over single objective ones, since provides a superior balance between cost and reliability indexes. The proposed planning approach can be useful tool for a practical distribution system planning.

\section{References}

1. Logrono, D.O., Wu, W.-F., Lu, Y.-A.: Multi-Objective Optimal Placement of Automatic Line Switches. In: Proceedings of the Institute of Industrial Engineers Asian Conference, pp. 471-478 (2013)

2. Ma, L., Lv, X., Wang, S., Miyajima, H.: Optimal switch placement in distribution networks under different conditions using improved GA. In: Second International Conference on Computational Intelligence and Natural Computing Proceedings (CINC), pp. 236-239 (2010)

3. Barroso, G.C., Leao, R.P.S.: Switch placement algorithm for reducing customers outage impacts on radial distribution networks. In: TENCON - IEEE Region 10 Conference (2012)

4. Moradi, A., Fotuhi-Firuzabad, M.: Optimal Switch Placement in Distribution Systems Using Trinary Particle Swarm Optimization Algorithm. IEEE Transactions on Power Delivery 23, 271-279 (2008)

5. Teng, J., Liu, Y.: A novel ACS-Based Optimum Switch Relocation Method. IEEE Transactions on Power Systems 18, 113-120 (2003)

6. Tippachon, W., Rerkpreedapong, D.: Multiobjective Optimal Placement of Switches and Protective Devices in Electric Distribution Systems using Ant Colony Optimation. Electric Power System Research 79, 1171-1178 (2009)

7. Silva, L.G.W., Pereira, R.A.F., Mantovani, J.R.S.: Opimized Placement of Control and Protective Devices in Electric Distribution Systems Through Reactive Tabu Search Algorithm. Electric Power System Research 78, 372-381 (2008)

8. Goroohi Sardou, I., Banejad, M., Hooshmand, R., Dastfan, A.: Modified shuffled frog leaping algorithm for optimal switch placement in distribution automation system using a multiobjective fuzzy approach. IET Generation, Transmission \& Distribution 6, 493-502 (2012)

9. Nascimento Alves, H.: A hybrid algorithm for optimal placement of switches devices in electric distribution systems. IEEE Latin America Transactions 10, 2118-2223 (2012)

10. Third Benchmarking Report on Quality of Electricity Supply - CEER - Council of European Energy Regulators. Technical report (2005)

11. Abido, M.A.: Environmental/economic power dispatch using multiobjective evolutionary algorithms. IEEE Transactions on Power Systems 18, 1529-1537 (2003)

12. Pires, D.F., Antunes, C.H., Martins, A.G.: NSGA-II with local search for a multi-objective reactive power compensation problem. International Journal of Electrical Power \& Energy Systems 43, 313-324 (2012) 